\title{
La Facoltà di Teologia nel rapporto verso le autorità ecclesiastiche alla luce della Costituzione Apostolica Sapientia Christiana ${ }^{1}$
}

\author{
Teološka fakulteta $v$ odnosu do cerkvenih oblasti \\ $v$ luči apostolske konstitucije Sapientia Christiana
}

Riassunto: La facoltà teologica è una istituzione di istruzione superiore scientifica, che può operare autonomamente all'interno della Chiesa oppure è inserita nell'università statale. A confronto delle altre facoltà di diverse discipline la sua natura è particolare in quanto partecipa della missione evangelizzatrice della Chiesa. La teologia è la scienza in cui si riflette la dottrina cattolica. Da questa premessa si deduce che solo la competente autorità ecclesiastica può istituirla o riconoscerla come tale, a prescindere dal fatto che operi solo all'interno della Chiesa o sia inserita nell'ambito dell'università statale. I docenti delle discipline teologiche hanno bisogno del relativo mandato della Chiesa per insegnare, che assicuri loro che l'insegnamento è conforme alla dottrina cattolica e all'insegnamento del Magistero della Chiesa e che sono in piena comunione con la Chiesa. Nel caso in cui la facoltà teologica sia inserita nell'università statale, vale sia la legislazione ecclesiastica che quella civile in materia; l'attività delle facoltà è in questo caso regolata in modo più precipuo dal Concordato o da altro accordo tra lo Stato e la Chiesa. Per l'istituzione e l'attività di una istituzione superiore svolgono un ruolo privilegiato la Santa Sede, la Conferenza Episcopale e il Gran Cancelliere, che è l'anello di congiunzione tra la stessa Facoltà e la Chiesa come ente che la erige.

Parole chiave: Facoltà di Teologia, università cattolica, teologia, Santa Sede, Gran Cancelliere, Conferenza Episcopale

Povzetek: Teološka fakulteta je znanstvena in vzgojna visokošolska ustanova, ki lahko deluje avtonomno znotraj Cerkve ali pa je vključena v državno univerzo. Njena narava je v primerjavi s preostalimi fakultetami drugih smeri posebna v tem, da sodeluje pri oznanjevalni službi Cerkve. Teologija je znanost, v kateri se zrcali katoliški nauk. Iz tega izhaja, da lahko teološko fakulteto ustanovi ali kot takšno prizna samo pristojna cerkvena oblast, ne glede

1 L'articolo è il risultato dell'attività del programma di ricerca P6-0262 Fonti giudeo-cristiane e ambiti della giustizia, finanziato dalla Agenzia Pubblica per l'attività di ricerca della Repubblica di Slovenia con i fondi pubblici. 
na to, ali deluje samo znotraj Cerkve ali pa je vključena v državno univerzo. Učitelji teoloških disciplin potrebujejo za poučevanje ustrezen mandat Cerkve, ki jim daje zagotovilo, da je njihovo učenje skladno s katoliškim naukom in učenjem cerkvenega učiteljstva in da so $v$ polnem občestvu s Cerkvijo. Če je teološka fakulteta vključena v državno univerzo, zanjo velja tako cerkvena kakor tudi civilna zakonodaja za to področje, delovanje fakultet pa v tem primeru natančneje ureja konkordat ali drug ustrezen sporazum med državo in Cerkvijo. Za ustanovitev in delovanje te visokošolske ustanove imajo posebno vlogo Sveti sedež, škofovska konferenca in veliki kancler, ki je vezni člen med fakulteto in Cerkvijo kot ustanoviteljem.

Ključne besede: Teološka fakulteta, katoliška univerza, teologija, Sveti sedež, veliki kancler, škofovska konferenca, Sveti sedež

\section{Introduzione}

La teologia cattolica e le istituzioni cattoliche di istruzione superiore occupano un posto importante nella formazione e nell'evangelizzazione della Chiesa. La loro missione è l'annuncio della buona novella del vangelo e la ricerca e l'approfondimento della verità. (Petkovšek 2018, 236; Slatinek 2017, 117) Pertanto non sorprende che a questo campo venga riservata una particolare attenzione anche nell'ordinamento giuridico autonomo della Chiesa Cattolica. Le norme fondamentali per l'attività delle facoltà teologiche in tutto il mondo sono regolate soprattutto dal Codice di Diritto Canonico del 1983 e dalla Costituzione Apostolica Sapientia christiana (SapCh) del 1979. Le facoltà teologiche e le scuole di istruzione teologica superiore possono operare in modo completamente autonomo nell'ambito della Chiesa, ma possono essere anche inserite nelle università statali, come testimonia una prassi frequente specialmente in Germania, Austria, Slovenia e Croazia. In Slovenia nel 2019 si celebra il Primo Centenario dell'Università di Ljubljana, di cui è membro fondatore anche la Facoltà di Teologia di Ljubljana, che dal 1968 ha una sede dislocata a Maribor. (Krajnc-Vrečko 2018, 15-20) Considerato che la Facoltà di Teologia dall'inizio fu parte integrante dell'università statale (Kolar 2010, 121-197), sebbene nel secondo dopoguerra il loro legame fosse interrotto per alcuni decenni per motivi ideologici, tuttavia la sua attività è regolata già dall'inizio anche dalla legislazione civile, oltre che dalle norme canoniche. ${ }^{2}$ A proposito dell'inserimento della Facoltà di Teologia

2 Come esempio di duplice autorità competente possiamo citare ad es. la nomina dei professori. Nel Regno di Serbi, Croati e Sloveni i professori della Facoltà di Teologia a Ljubljana erano nominati dal Ministro per l'istruzione di Belgrado su proposta della stessa Facoltà. Cf. Zapisnik seje fakultetnega sveta 19. 9. 1919. ATF, šk. 4. Redni fakultetni svet 1919-1970. (Saje 2019, 2-4) 
tra le istituzioni statali di istruzione superiore si parla dal punto di vista giuridico di un ambito misto di competenze, laddove per l'attività e il governo della Facoltà è indispensabile la collaborazione sia delle strutture di governo statali che di quelle ecclesiastiche. A livello di Chiesa universale quest'ultima è rappresentata dalla Santa Sede come autorità suprema, mentre a livello locale riveste un ruolo particolare il Gran Cancelliere della Facoltà di Teologia ossia l'Ordinario del luogo. Come nella legislazione civile, che regola le istituzioni statali di istruzione superiore, anche quella canonica regola le questioni relative ai contenuti e ai soggetti, nella qual cosa entrambi i rami competenti di governo, sia ecclesiastico che civile, sono consapevoli delle proprie competenze e limitazioni, rispettandosi l'uno l'altro e risolvendo le questioni in comune in spirito di collaborazione e tolleranza per il bene della collettività. (Sporazum med Republiko Slovenijo in Svetim sedežem 2004, art. 1, 14)

\section{Differenza tra università e facoltà cattoliche ed ecclesiastiche}

Rispetto alle finalità e agli obiettivi distinguiamo l'istruzione ecclesiastica superiore in due tipi: il legislatore supremo determina norme particolari anzitutto per le università cattoliche, in particolare con la Costituzione Apostolica Ex corde ecclesiae (ECE) del 1990, laddove si possono istituire corsi di studi con i quali la Chiesa contribuisce ad una maggiore cultura e al perfezionamento della persona. (Ammer 1994, 15-22; Krämer 1991, 25-47) In questa sede non si pongono limiti più precisi rispetto agli indirizzi di studio. (CIC, can. 807-814; ECE, 1475-1509) È necessario evidenziare che il diritto della Chiesa di istituire centri di istruzione superiore non si fonda solo sul principio fondamentale del suo diritto di istituire scuole in generale (CIC, can. 800), ma ha un significato più ampio. (Leskovar 2002, 677-692) L'università cattolica, come ogni altra università, è finalizzata alla ricerca, al progresso scientifico e all'approfondimento dello spirito e della cultura dell'uomo. (ECE II, 2 \$) In quanto cattolica, l'università ha alcune specifiche peculiarità, come l'antropologia cristiana, la ricerca intessuta di fede cattolica, la diaconia alla persona, alla comunione e alla comunità cristiana. (ECE I, 13; II, 2 \3) La finalità delle università cattoliche è l'armonia e la collaborazione di ricerca e scienza con il messaggio cristiano, per cui la fede e la scienza non si escludono, in accordo con il Magistero conciliare fondato sulla dottrina tomistica. (GS 36) 
L'altro tipo di istituzioni di istruzione superiore nella Chiesa è rappresentato dalle università e facoltà ecclesiastiche. A differenza del CIC del 1917, il nuovo Codice distingue chiaramente tra università e facoltà cattoliche ed ecclesiastiche. (CIC, can. 815-821) Mentre le università cattoliche, oltre alle norme generali del CIC, sono anche oggetto della ECE, le università e facoltà ecclesiastiche sono più propriamente definite dalla Costituzione Apostolica Sapientia christiana (SapCh) del 15 aprile 1979, a cui sono seguiti ancora altri documenti della Congregazione per la scuola e l'istruzione, per es. del 29 aprile 1979 (Ordinationes). Secondo il processo di Bologna e dell'istruzione superiore e le relative trasformazioni da esso determinate per le facoltà teologiche, la Congregazione per la scuola e l'istruzione ha pubblicato una serie di istruzioni e decreti. (Shmitz e Rhode 2011, 3454) La suddetta Costituzione Apostolica riporta questa definizione delle università e facoltà ecclesiastiche: „Università e facoltà ecclesiastiche sono dette quelle che, canonicamente erette o approvate dalla Sede Apostolica, coltivano e insegnano la dottrina sacra e le scienze con essa collegate, fruendo del diritto di conferire i gradi accademici per autorità della Santa Sede." (SapCh, art. 2) La loro finalità è la ricerca delle discipline teologiche e delle scienze sacre con essa collegate (SapCh, art. 3; CIC, can. 815), l'istruzione e la diffusione dell'evangelizzazione in collaborazione con la suprema gerarchia ecclesiastica e le Chiese locali. (SapCh, art. 3 \$3) L'oggetto dell'insegnamento e della ricerca sotto la direzione della Santa Sede è direttamente collegato al deposito della fede; per l'organizzazione degli studi teologici la competenza ricade sulla Chiesa stessa. (Rhode e Rüfner 2012, 20) Tra le discipline importanti che si insegnano nelle facoltà teologiche si annoverano la filosofia, la teologia, il diritto canonico, ambiti esplicitamente citati anche dalla Costituzione Apostolica sull'argomento. (SapCh, art. 66-83) La facoltà teologica di regola conferisce titoli accademici solo nel campo della teologia, ma con l'autorizzazione della Santa Sede può conferire anche titoli di altri indirizzi di studio. Nella Chiesa hanno effetto giuridico solo quei titoli accademici (CIC, can. 817) conferiti dalle facoltà teologiche o da altre istituzioni di istruzione superiore all'interno della Chiesa o da quelle integrate nelle università statali, per le quali la Santa Sede ha conferito il mandato di articolare singoli programmi, oppure da istituzioni scientifiche e accademiche già esistenti, che la Santa Sede ha confermato come tali. 
Mentre la maggior parte delle università ecclesiastiche sono a Roma e si chiamano università pontificie, le facoltà teologiche nella maggioranza dei casi sono diffuse in tutto il mondo in piena autonomia della Chiesa, altre - come ad es. la Facoltà di Teologia di Ljubljana - sono parte integrante dell'università statale. Indipendentemente dal fatto che la facoltà teologica sia completamente autonoma nell'ambito della Chiesa ovvero parte di una università statale, la Chiesa Cattolica si riserva il diritto di erezione e guida, di nomina dei professori e di controllo, non solo per il suo interesse a controllare o entrare in questioni statali, quanto piuttosto per il semplice fatto che la teologia cattolica può essere insegnata correttamente solo in un forte legame con la Chiesa Cattolica. (Schmitz e Rhode 2011, 20)

A questo punto lasciamo da parte le università ecclesiastiche o pontificie, per le quali vigono determinati statuti oltre alla legislazione del CIC, per occuparci solo delle facoltà teologiche e del loro rapporto con il governo della Chiesa alla luce di SapCh e nel rispetto delle competenze dello Stato, qualora si tratti di una facoltà che è parte di una università statale.

\section{Particolarità delle facoltà teologiche nelle università statali}

La Teologia rientra tra i più antichi indirizzi di studio universitario. Prima che nascesse un sistema di istruzione superiore organizzato, si studiava specialmente nelle scuole dei monasteri. Con l'istituzione delle moderne università europee le facoltà teologiche sono diventate un importante partner di dialogo e collaborazione nell'ambito delle discipline umanistiche e della cultura, in modo particolare nelle terre germanofone ovvero negli Stati che storicamente appartenevano alla monarchia austro-ungarica. La facoltà teologica in una università statale completa lo spettro degli indirizzi di studio, rappresentando un arricchimento per la società e un contributo allo sviluppo delle scienze umanistiche, del pensiero filosofico e della cultura generale. ${ }^{3}$ Per l'attività di queste facoltà, nel contesto dello

3 In modo simile anche i licei cattolici sloveni, soprattutto con l'insegnamento di Fede e cultura, contribuiscono alla conoscenza della cultura, della convivenza e del dialogo interreligioso. »Pri religijskem pouku v šoli ne govorimo o katehezi, torej o uvajanju v krščanstvo, temveč o razlaganju krščanskega pogleda na svet in pogleda tudi drugih religij; to dijakom omogoča, da prepoznajo pomen religij za osebno in družbeno življenje ter zgodovino našega naroda in vseh civilizacij." (Globokar e Rifel 2017, 358) 
studio universitario in generale, è necessario una adeguata intesa a livello di accordo internazionale tra la Santa Sede e il singolo Stato, di solito sotto forma di concordato o di un altro accordo giuridicamente valido. ${ }^{4}$ Il prerequisito per stipulare un tale accordo è in primo luogo il riconoscimento - da parte dello Stato - della Chiesa come soggetto giuridico indipendente e autonomo e, in secondo luogo, della Teologia come libera disciplina scientifica e pertanto importante per la comprensione e lo sviluppo del mondo. La Teologia come dottrina su Dio e disciplina sulla Parola di Dio incarnata di conseguenza, per sua stessa natura, è possibile solo sotto la guida del Magistero della Chiesa. (May 1969, 291-309) L'ordinamento giuridico della Chiesa si intreccia con quello statale in maniera evidente a proposito della nomina dei professori, ad esempio (CIC, can. 812), ma anche nella disciplina che regola il riconoscimento dei titoli scientifici e i requisiti per l'iscrizione, che devono essere giuridicamente sullo stesso livello delle altre facoltà. ${ }^{5}$ Per l'insegnamento delle discipline teologiche in una qualunque istituzione di istruzione superiore - aldilà se questa sia autonoma o inserita nell'università statale - è necessario avere prioritariamente il permesso della Chiesa di insegnare le sacre scienze e il Nihil obstat ${ }^{6}$ che attesta l'assenza di impedimenti. L'attività delle facoltà teologiche a livello di Chiesa universale è definita dalla già citata Costituzione Apostolica SapCh del 1979, che regola soprattutto i seguenti ambiti: la natura e la missione delle scuole ecclesiastiche superiori, la struttura della comunità accademica, il collegio dei professori, gli studenti, l'organizzazione e il piano degli studi, i titoli accademici, i sussidi didattici, i mezzi economici per l'attività, la collaborazione tra le facoltà. La Congregazione per l'educazione e l'istruzione con il permesso del Papa ha integrato la suddetta Costituzione due volte, anzitutto (2002) nell'art.

4 Nella Repubblica di Slovenia il documento fondamentale per inserire la Facoltà di Teologia nell'ambito dell'Università di Ljubljana è rappresentato dall'Accordo tra la RS e la Santa Sede sulle questioni giuridiche all'art. 10. »La Chiesa Cattolica in conformità alla legislazione della Repubblica di Slovenia e al Diritto Canonico ha diritto di istituire e amministrare scuole di ogni ordine e grado, convitti per la scuola primaria e secondaria e altre istituzioni di istruzione e formazione."

5 "Wichtig bleibt immer die Gleichwertigkeit des Studiums. Deshalb müssen auch in kirchlichen Hochschulen die Lehraufgaben von hauptberuflich Lehrenden wahrgenommen werden, deren Qualifikation der von Lehrenden an staatlichen Hochschulen entspricht. Die Studierenden müssen wie die an staatlichen Hochschulen vorgebildet sein.« (Schmitz e Rhode 2011, 20)

6 "Mit der Erteilung oder Versagung des Nihil obstat vermag die katholische Kirche den Eintritt von Geistlichen in weltlicher Ämter, im Staat sowie in den Gemeinden und in der anderen Körperschaften des öffentlichen Rechts, zu regulieren.«(Weber 1939, 193) 
76 circa lo studio del Diritto Canonico e successivamente (2011) circa lo studio della Filosofia nell'art. 72, 81 e 83.

In seguito alla decisione della Santa Sede di entrare e collaborare nel processo di Bologna nel 2003, la Congregazione per l'educazione e l'istruzione ha pubblicato una serie di decreti e istruzioni, ${ }^{7}$ indirizzate ai gran cancellieri delle facoltà, ai rettori, ai decani e ai presidenti delle Conferenze Episcopali. In conformità al can. 34 del CIC possiamo comprendere le suddette istruzioni in senso simile alle Ordinationes della Congregazione per l'educazione allegate alla Costituzione Apostolica SapCh (1979), in quanto le regole normative vigenti chiariscono, spiegano e definiscono la modalità obbligatoria del loro uso. Sono pubblicate ad uso di quanti devono aver cura di applicare le leggi. (Hallermann 2007, 500-505)

\section{Rapporto delle facoltà teologiche verso l'autorità ecclesiastica}

La Teologia è una disciplina scientifica. Come scienza rispetta i consolidati metodi di ricerca, cerca la verità su Dio, sul mondo e sull'uomo. (Tommasi 2018, 210) Si fonda sulla rivelazione divina, sulla Sacra Scrittura e sulla dottrina del Magistero della Chiesa, che insieme con il Papa spiega in maniera autentica la dottrina. La Chiesa, a cui il Signore Gesù ha affidato il deposito della fede perché riconosca con l'aiuto dello Spirito Santo la verità rivelata, custodisce, ricerca, annuncia e spiega questo deposito. Ha il diritto indipendente e naturale di annunciare il Vangelo a tutti gli uomini. (CIC, can. 747) In conformità con SapCh il compito delle facoltà teologiche è promuovere la ricerca delle sacre scienze, soprattutto sulla base della rivelazione cristiana, valorizzare le conoscenze acquisite alla luce delle sfide del mondo moderno e metterle a disposizione della gente nei diversi ambienti culturali. (art. 3) La facoltà teologica in questo senso svolge un servizio di evangelizzazione, pertanto deve essere in stretto legame e unione con la guida gerarchica della Chiesa, perché possa custodire intatto il depositum fidei e il magisterium authenticum. (Schmitz e Rhode 2011, 55) Bisogna evidenziare a questo punto che le facoltà come istituzioni

7 I documenti sono accessibili sul sito web della Santa Sede: http://www.educatio.va/content/cec/it/ documentazione-e-materiali/documenti-della-congregazione.html (accesso 28. aprile 2019). 
di istruzione superiore godono al contempo di propria autonomia e libertà di azione. Allo stesso modo è assicurata la libertà dei professori, che si dedicano alle sacre scienze nei campi per cui sono specializzati nel dovuto rispetto del Magistero della Chiesa, che è l'unica importante limitazione. (GS, art. 59; SapCh, art. 39; CIC, can. 218) La libertà della ricerca è limitata dalla stessa rivelazione e dalla sua autentica interpretazione. La Chiesa si riserva il diritto di nominare i professori di Teologia che per la loro missione di insegnamento hanno bisogno della nomina dell'autorità ecclesiastica competente (mandatum). (Kasper 1982, 13-44; Pfeifer 1981, 207-223)

Il Magistero ecclesiastico autentico, oltre alla competenza di nominare i professori e promuovere lo sviluppo delle discipline teologiche, svolge anche il compito di controllo sull'insegnamento della Teologia per impedire in qualche modo la diffusione di spiegazioni errate. L'interpretazione autentica e l'insegnamento teologico accademico nella Chiesa e per la Chiesa hanno una funzione indipendente e separata. Il Magistero non è un organismo di ricerca, pertanto non possiede un sapere specialistico. La professione di un docente di Teologia non è parallela o alternativa accanto al Magistero della Chiesa. Tanto il Magistero della Chiesa quanto i professori di Teologia hanno ognuno la propria specificità e responsabilità, entrambi lavorano per la crescita del Regno di Dio e si completano e arricchiscono a vicenda. (Schmitz e Rhode 2011, 60) In questo senso è necessario accennare alle più importanti istituzioni ecclesiastiche che hanno un compito particolarmente rilevante e responsabile nel rapporto verso le facoltà teologiche, nella fattispecie la Santa Sede, le Conferenze Episcopali, il Gran Cancelliere e l'Ordinario del luogo.

\subsection{La Santa Sede}

Quando parliamo della massima autorità della Chiesa, utilizziamo il termine Santa Sede o Sede Apostolica, non alludendo solo al Romano Pontefice come pastore supremo della Chiesa, ma piuttosto all'intera Curia romana ${ }^{8}$

8 "La Curia romana è l'insieme dei dicasteri e degli organismi che coadiuvano il Romano Pontefice nell'esercizio del suo supremo ufficio pastorale per il bene e il servizio della Chiesa universale e le Chiese particolari, esercizio con il quale si rafforzano l'unità di fede e la comunione del popolo di Dio e si promuove la missione propria della Chiesa nel mondo. (PB, art. 1) 
con i suoi uffici, che sotto l'autorità pontificia svolge il suo servizio per il bene delle Chiese locali. (CIC, can. 360, 361) La Costituzione Apostolica Pastor bonus regola la sua attività. (Giovanni Paolo II 1988) La Curia romana non gode di costituzione divina, né va equiparata all'Assemblea dei vescovi. Opera in quanto è in relazione con il ministero petrino del Papa. Non ha alcun potere all'infuori di quelli ricevuti dal Romano Pontefice (CIC, can. 131 』2) e dispone perciò di potestà vicaria. (De Paolis 2003, 177-183) Per l'attività delle facoltà teologiche tra i Dicasteri è competente la Congregazione per l'educazione e l'istruzione. La Santa Sede in questo senso è competente anzitutto per erigere e riconoscere le facoltà teologiche e il loro governo (CIC, can. $816 \S 1$; SapCh, art. 2, 5, 61), per approvare gli Statuti e il piano di studio (CIC, can. $816 \$ 2$; SapCh, art. 7), nominare il rettore o il presidente (SapCh, art. 18), concedere ai docenti il permesso di insegnare (SapCh, art. 18), progettare l'istituzione di nuove facoltà (SapCh, art. 60), collegare gli istituti con le altre facoltà (SapCh, art. 62, 63), raccogliere le statistiche annuali e le relazioni triennali sull'attività svolta (OrdSapCh, art. 8, 6; 14,6 $6^{\circ}$, concedere il permesso del Nihil obstat e attribuire il dottorato onorario. (OrdSapCh, art. 38) Queste regole generali non valgono del tutto per le facoltà teologiche tedesche, laddove sono state concesse determinate eccezioni da parte dell'autorità competente. (Schmitz e Rhode 2011, 61-62) È qui necessario accennare anche al fatto che la Santa Sede per principio non riconosce i gradi accademici dello studio teologico nelle facoltà e nelle scuole superiori che non sono state riconosciute da parte dell'autorità ecclesiastica competente. (SapCh, art. 9)

\subsection{Le competenze delle Conferenze Episcopali}

La Conferenza Episcopale per sua natura è un'assemblea permanente di vescovi di un singolo popolo o territorio e svolge alcuni compiti pastorali per il bene dei fedeli. (CIC, can. 447) Ha autorità legislativa regolare e propria, tuttavia solo nei casi di pubblicazione dei suddetti decreti generali, come stabilisce il diritto generale o la Sede Apostolica. I suoi decreti sono redatti all'interno e sulla base delle leggi generali già vigenti e ad essa superiori, a cui non aggiungono né sottraggono nulla. Le sue norme di conseguenza non sono poste a livello di leggi. Negli altri casi la Conferenza Episcopale non ha potere legislativo (CIC, can. 119, 3을, ma il suo presidente o la stessa Conferenza possono validamente assumere delle decisioni solo nel caso in cui tutti i singoli membri esprimano 
il consenso sulle singole decisioni. (Saje 2015, 125) A proposito della competenza della Conferenza Episcopale nell'ambito dell'istruzione superiore, il suo compito fondamentale consiste nel controllare l'attività delle facoltà teologiche insieme con i vescovi del luogo e nel curare che vi si insegni la dottrina cattolica in conformità con il Magistero della Chiesa. (CIC, can. 818; OrdSapCh, art. 5, 1) Rispetto al grande significato delle facoltà teologiche per la vita della Chiesa, il suo compito è promuovere queste istituzioni di istruzione superiore e assicurare le condizioni per il loro progresso e la progettazione di nuove facoltà. (SapCh, art. 4, 60, 61) Per quanto attiene all'istruzione e alla formazione dei sacerdoti, che non si svolgono all'interno dei seminari, ma nelle scuole teologiche ecclesiastiche all'interno della Chiesa ovvero all'esterno in università statali, la Conferenza Episcopale ha il compito di definire le condizioni circa l'istruzione filosofica e teologica e i coerenti insegnamenti di questo campo in riferimento alle necessità pastorali di una determinata Chiesa locale. (CIC, can. 242) Il diritto generale conferisce alla Conferenza Episcopale competenze specifiche non solo nell'ambito interno alla Chiesa, ma anche per coloro che operano nel contesto delle università statali, soprattutto in merito alla progettazione degli studi teologici, alla definizione dei criteri minimi e dello stesso modo di articolare lo studio, competenze che di solito sono definite più concretamente nei concordati o negli altri accordi internazionali. (Schmitz e Rhode 2011, 64)

\subsection{Il ruolo del Gran Cancelliere}

La missione fondamentale e il compito delle facoltà teologiche sono l'insegnamento, la ricerca e la collaborazione nel ministero di evangelizzazione della Chiesa nel più ampio senso della parola. La sua tipica missione è l'istruzione dei candidati al sacerdozio, la qual cosa non rappresenta una condizione assoluta in vista della loro esistenza e attività. (CIC, can. 819; SapCh, art. 74 \ 1) Lo Statuto della facoltà, che regola l'intera attività dell'istituzione accademica, del programma di studi, degli studenti, dei professori, del governo della facoltà e del conferimento dei titoli accademici, deve essere approvato dalla Santa Sede. (CIC, can. 816; SapCh, 16, 23, $25 \rrbracket 3,32 \rrbracket 1$; OrdSapCh, 11, $16 \rrbracket 1$ ) Il Gran Cancelliere (Magnus Cancellarius) in conformità con l'ordinamento giuridico per l'istruzione superiore ecclesiastica rappresenta la prima e diretta autorità della Chiesa per l'università o la facoltà teologica ecclesiastica. (Hommens 1985, 15-20) 
Non può intervenire direttamente nell'attività dell'istituzione, ma almeno indirettamente ha alcune funzioni e competenze particolari per l'attività della Conferenza Episcopale. È l'anello di congiunzione tra la Sede Apostolica e la comunità accademica della facoltà, rappresenta la Santa Sede nel rapporto con il governo della facoltà, mentre nel rapporto verso la Santa Sede rappresenta gli interessi della facoltà e tratta i problemi attuali e le questioni aperte. (SapCh, art. 12) A proposito dei compiti particolari del Gran Cancelliere è opportuno considerare nello specifico i seguenti uffici: cura del progresso della facoltà, promozione della ricerca e dell'attività scientifica in conformità con la dottrina cattolica, l'insegnamento del Magistero della Chiesa e le regole giuridiche vigenti, promozione della collaborazione tra i membri della facoltà ossia la comunità accademica, richiesta dei permessi per l'insegnamento ai singoli docenti presso la Congregazione per l'educazione e l'istruzione (nihil obstat), rito del giuramento e della professione di fede del rettore o presidente, conferimento della missione canonica ai singoli docenti (missio canonica), informazione alla Santa Sede sulle attività più importanti della facoltà e invio della relazione triennale sul lavoro della stessa facoltà. (OrdSapCh, art. 8) Il compito del Gran Cancelliere in senso generale consiste nel promuovere l'attività scientifica e il pensiero e la cultura cristiana, oltre che nell'impegno per l'integrazione della facoltà nella vita della Chiesa locale e nella società. Tra le sue responsabilità si annovera anche il ruolo di mediatore nei casi di conflitto e l'attenzione all'insegnamento della dottrina cattolica in sintonia con la tradizione e il Magistero della Chiesa. (SapCh, art. 70)

\section{Conclusione}

Le facoltà teologiche o le altre istituzioni d'ecclesiastiche di istruzione superiore contribuiscono alla missione evangelizzatrice della Chiesa. Di conseguenza anche i docenti di queste scuole non insegnano le discipline teologiche come privati, ma in nome della Chiesa. Istruiscono e formano i futuri sacerdoti e gli altri teologi laici. Per la Chiesa Cattolica è peculiare il Magistero ordinato gerarchicamente, come autentici maestri sono definiti il Papa e l'Assemblea dei vescovi. I professori di Teologia, sebbene abbiano idonei titoli accademici, possono insegnare solo con l'esplicito permesso dell'autorità competente. L'autorità suprema non esige da loro solo le competenze scientifiche o specialistiche, ma anche la fedeltà alla 
dottrina autentica. La ragione consiste nel fatto che il loro lavoro è al contempo missione della Chiesa per l'evangelizzazione, operano in suo nome, di conseguenza necessitano di un particolare mandato per insegnare le sacre scienze. (CIC, can 812) Tutti i professori di Teologia necessitano del mandato per l'insegnamento nelle facoltà teologiche e in tutte le altre scuole di istruzione superiore collegate con la Chiesa, in cui si insegnano le discipline teologiche. (Montini 1992, 79-109)

Nell'attività di ricerca il legislatore supremo assicura loro la libertà necessaria nei campi in cui sono specializzati, con il dovuto rispetto verso la dottrina cattolica e l'insegnamento del Magistero della Chiesa. (CIC, can. 218) Come tutti i fedeli sono tenuti a credere a tutto ciò che riguarda la Parola di Dio scritta e tramandata e a ciò che il Magistero della Chiesa propone di credere nel Magistero sia straordinario che ordinario della Chiesa. (CIC, can. 750) Allo stesso modo sono tenuti ad recepire ciò che il Magistero propone definitivamente di credere, affinché il deposito della fede sia custodito fedelmente. Tutto ciò vale esplicitamente per i professori di Teologia che insegnano nelle istituzioni ecclesiastiche di istruzione superiore con la differenza che si esige da loro anche una vita esemplare e meritevole di lode nella dottrina autentica. (CIC, can. 810; 833 \6) Prima di intraprendere il loro lavoro devono professare la fede davanti ai vescovi del luogo e giurare fedeltà. (Malvaux 2003, 538) Devono esplicitamente concordare con l'insegnamento del Pontefice e dell'autentico Magistero della Chiesa, in caso contrario non si conferisce loro il permesso di insegnare le scienze teologiche; nei casi più gravi, se insegnassero in contrasto con la dottrina della Chiesa, si sospende loro il mandato già conferito. 


\section{Sigle e abbreviazioni}

$\begin{array}{ll}\text { AAS } & \text { Acta Apostolicae Sedis } \\ \text { AES } & \text { Acta Ecclesiastica Sloveniae } \\ \text { AfkKR } & \text { Archiv für Katholisches Kirchenrecht } \\ \text { ATF } & \text { Arhiv Teološke fakultete } \\ \text { BV } & \text { Bogoslovni vestnik } \\ \text { ECE } & \text { Costituzione Apostolica Ex corde Ecclesiae } \\ \text { GS } & \text { Costituzione Pastorale del Concilio Vaticano II Gaudium et spes } \\ \text { OrdSapCh } & \text { "Ordinationes« alla Costituzione Apostolica Sapientia Christiana } \\ \text { PB } & \text { Costituzione Apostolica Pastor bonus } \\ \text { SapCh } & \text { Costituzione Apostolica Sapientia Christiana } \\ \text { CIC } & \text { Codex Iuris Canonici } \\ \text { TThZ } & \text { Trierer Theologische Zeitschrift }\end{array}$

\section{Fonti}

Benedetto XV. 2017. Codex Iuris Canonici, AAS 9/II: 1-456.

Codice di Diritto canonico commentato. 2017. Milano: Ancora.

Concilio Vaticano II. 1966. Costituzione Pastorale Gaudium et spes. 7 dicembre. AAS 58: 1025-1115.

\section{Congregazione per l'Educazione Cattolica.} 1979. »Ordinationes« alla Costituzione Apostolica Sapientia Christiana. AAS 71: 500-521.

- - . 2003. Decreto Novo Codice del 2. 9. 2002. AAS 95: 281-285.

- - -. 2011. Decreto di riforma degli studi ecclesiastici di filosofia del 28. 1. 2011. http://www.vatican.va/roman_curia/ congregations/ccatheduc/documents/ rc_con_ccatheduc_doc_20110128_dec-rif-filosofia_it.html (accesso 25 aprile 2019).

Deutsche Bischofskonferenz. 2011.

Katholische Theologie und Kirchliches Hochschulrecht. Arbeitshilfen Nr. 100. Bonn: Sekretariat der deutschen Bischofskonferenz.
Giovanni Paolo II. 1983. Codex Iuris Canonici. AAS 75/II: 1-317.

- - - 1988. Costituzione Apostolica Pastor bonus, 28 giugno. AAS 80: 841-930.

- - -. 1990. Costituzione Apostolica Ex corde Ecclesiae, 15 agosto. AAS 82: 1475-1509.

- - -. 1979. Costituzione Apostolica Sapientia Christiana. 15 aprile. AAS 71: 469-499.

Koncilski odloki. 1980. Konstitucije, odloki, izjave, poslanice 2 . vatikanskega vesoljnega cerkvenega zbora (1962-1965). Ljubljana: Nadškofijski ordinariat.

Sporazum med Republiko Slovenijo in Svetim sedežem. 6. februar 2004. http://www. uradni-list.si/glasilo-uradni-list-rs/vsebina/47165 (accesso 1 maggio 2019).

Zapisnik seje fakultetnega sveta, 19. 9. 1919. In: $A T F$, šk. 4. Redni fakultetni svet 1919-1970. 


\section{Studi}

Ammer, Josef. 1994. Zum Recht der »katholischen Universität:: Genese und Exegese der apostolischen Konstitution "Ex corde Ecclesiae"vom 15. August 1990, Würzburg: Echter Verlag.

De Paolis, Velasio. 2003. La Curia Romana a servizio della Chiesa. In: Chiese particolari e la Chiesa universale. Milano: Glossa.

Globokar, Roman e Tadej Rifel. 2017. Medverski dialog pri religijskem pouku v Sloveniji. Bogoslovni vestnik 77: 357-368

Hallermann, Heribert. 2007. Ein neues kirchliches Hochschulrecht? In: In Dienst von Kirche und Wissenschaft, 500-505. Ed. Wilhelm Rees, Sabine Demel, Ludger Müller. Berlin: Duncker und Humbolt.

Hommens, Maximilian. 1985. Magnus Cancelarius einer kirchlichen Hochschule. St. Ottilien: Eos Verlag.

Kasper, Walter. 1982. Wissenschaftliche Freiheit und lehramtliche Bindung der katholischen Theologie. In: Essener Gespräche zum Thema Staat und Kirche, Teil 16, 12-42. Munster: Aschendorff.

Kolar, Bogdan. 2010. Teološka fakulteta - del univerze v Ljubljani. AES 32: 121-197.

Krajnc-Vrečko, Fanika. 2018. 50 let enote Teološke fakultete v Mariboru (19682018). Edinost in dialog 73: 15-20.

Krämer, Peter. 1991. Die katholische Universität. Kirchenrechtliche Perspektiven. AfkKR 160: 25-47.

Leskovar, Mitja. 2002. Univerza - katoliška ali navadna?. Bogoslovni vestnik 63: 677-692.

Malvaux, Benoît. 2003. Les professeurs et la mission canonique, nihil obstat, mandat d'enseigner, profession de foi, serment de fidélité. Studia Canonica 37: 521-548.

May, Georg. 1969. Die Funktion der Theologie in Kirche und Gesellschaft. In: Paul Neuenzeit, ed. Die Funktion der Theologie in Kirche und Gesellschaft, 291-309. München: Kösel Verlag.

Edinost in dialog 74 (2019) 1: 239-252
Montini, Gian Paolo. 1922. La vocazione all'insegnamento nella Chiesa: dalla missione al mandato. In: Libertà e obbedienza nella Chiesa, 79-109. Brescia: Morcelliana.

Petkovšek, Robert. 2018. Veselje resnice in svetopisemski monoteizem. Edinost in dialog 73: 235-258.

Pfeifer, Helmut. 1981. Theologie und Lehramt. TThZ 90: 207-223.

Rhode, Ulrich e Wolfgang Rüfner. 2012. Kirchliche Hochschulen. Göttingen: De Gruyter.

Saje, Andrej. 2015. Normativna aktivnost biskupskih konferencija i krajevnih sabora. In: Zakon u životu partikularne Cerkve, 125-138. Zagreb: Glas koncila.

- - -. 2019. Gregorij Rožman in njegov prispevek k razvoju cerkvenega prava in Teološke fakultete v Ljubljani. AES 41: 1-18, in stampa.

Schmitz, Heribert e Ulrich Rhode. 2011. Einführung. In: Katholische Theologie und Kirchliches Hochschulrecht, Arbeitshilfen Nr. 100, 19-186. Ur. Deutsche Bischofskonferenz. Bonn: Sekretariat der deutschen Bischofskonferenz.

Slatinek, Stanislav. 2017. Odpustki - izhodiščna točka reformacije. Edinost in dialog 72: 113-125.

Tommasi, Roberto. 2018. Veritatis gaudium, impulso per una teologia sulla frontiera. Edinost in dialog 73: 207-223.

Weber, Werner. 1939. Das Nihil obstat. Beitrage zum Verhältnis von Staat und katholischer Kirche. Zeitschrift für die gesamte Staatswissenschaft 99: 193-244. 